\title{
Moderne Parodontaltherapie: Mehr als Kürettage
}

\begin{abstract}
In der Behandlung von parodontalen Erkrankungen gibt es viele moderne Möglichkeiten der Therapie. Im Bewertungsmaßstab zahnärztlicher Leistungen (BEMA) existiert allerdings eine Reihe von Leistungsausschlüssen. Der nachfolgende Text zeigt die Möglichkeiten der Privatliquidation von

Parodontalbehandlungen mit Versicherten der Gesetzlichen Krankenversicherung (GKV) auf.
\end{abstract}

Die plastischen Parodontaltherapien, wie die Behandlung der Rezessionen, des Fehlens keratinisierter Gingiva und der verkürzten angewachsenen Schleimhaut, gehören nicht zur vertragszahnärztlichen Versorgung der GKV-Versicherten. Werden diese Behandlungsmaßnahmen beim GKV-Patienten nach entsprechenden Vereinbarungen privat berechnet, so kommen hierfür die GOZ-Nr. 4120 „Verlegen eines gestielten Schleimhautlappens, je Kieferhälfte oder Frontzahnbereich“, die GOZNr. 4130 „Gewinnung und Transplantation von Schleimhaut, gegebenenfalls einschließlich Versorgung der Entnahmestelle, je Transplantat“ oder die GOZ-Nr. 4133 „Gewinnung und Transplantation von Bindegewebe einschließlich Versorgung der Entnahmestelle, je Zahnzwischenraum“ zum Einsatz.

\section{Rekonstruktive Maßnahmen sind Privatleistung}

Genauso sind alle rekonstruktiven oder regenerativen Maßnahmen am knöchernen parodontalen Hartgewebe nicht Leistungsinhalt der GKV. Hierzu gehören die Implantation von Knochen oder Knochenersatzmaterialien, die gesteuerte Geweberegeneration (GTR), die Anwendung von Wachstumsfaktoren und Matrixproteinen oder Kombinationen dieser Maßnahmen. Diese Maßnahmen werden privat nach der GOZ-Nr. 4110 „Auffüllen von parodontalen Knochendefekten mit Aufbaumaterial (Knochen- und/ oder Knochenersatzmaterial), auch Einbringen von Proteinen zur regenerativen Behandlung parodontaler Defekte, gegebenenfalls einschließlich Materialentnahme im Aufbaugebiet, je Zahn oder Parodontium oder Implantat“ und der GOZ-Nr. 4138 „Verwendung einer Membran zur Behandlung eines Knochendefektes einschließlich Fixierung, je Zahn, je Implantat" berechnet. Die Entfernung einer GTR-Membran wird nach der GOZ-Nr. 9160 berechnet. Für weitergehende Maßnahmen kommen auch Gebührennummern aus dem Kapitel K in der GOZ oder der GOÄ in Frage.

Aber auch die resektive Osteoplastik/Tunnelierung beziehungsweise klinische Kronenverlängerung ist nicht im BEMA enthalten. Wird diese Maßnahme vom GKV-Versicherten zum Zahnerhalt gewünscht, so kann die GOZ-Nr. 4136 vereinbart werden, allerdings nicht sitzungsgleich mit den BEMA-Nummern P202/P203 (offene PAR-Therapie).

Bereits in der parodontologischen Diagnostik trifft man auf Leistungsausschlüsse, so zum Beispiel die mikrobiologische Diagnostik oder Gensonden- beziehungsweise enzymatische Tests. Für die Entnahme von Abstrichen steht privatzahnärztlich die GOÄ-Nr. 298 - je Entnahmestelle - zur Verfügung. Moderne, aussagefähige Tests werden in aller Regel von speziellen mikrobiologischen Labors durchgeführt und auch von diesen Labors

\section{Ausschluss medikamentöser Therapien}

Neben modernen chirurgischen Therapien sind auch bestimmte medikamentöse Therapien in der GKV ausgeschlossen, so zum Beispiel die lokale Antibiotikatherapie (Chlorhexamed-Zubereitungen wie Periochip, CHX-Gel oder Doxycyclin-Gel etc.) und die "full mouth disinfection" (FMD). Berechnet werden die lokal antibakteriellen Maßnahmen nach der GOZNr. 4025 „Subgingivale medikamentöse antibakterielle Lokalapplikation, je Zahn", wobei hier die verwendeten antibakteriellen Materialien gesondert berechnungsfähig sind. Die FMD wird, da nicht in der GOZ 2012 enthalten, gemäß §6,1 GOZ analog berechnet.

berechnet. Die Fremdrechnungen dieser Testungen werden an den Patienten direkt weitergereicht.

Unter die privat vereinbarungsfähigen modernen selbstständigen Behandlungsmaßnahmen fallen auch alle diejenigen, die in der GOZ 2012 nicht in eigenen Ziffern enthalten sind. Neben der FMD sind dies zum Beispiel die photodynamische Therapie (PT) oder Deepithelisierungsmaßnahmen mit Hilfe von Laserlicht. Da es sich hier um selbstständige Behandlungsmaßnahmen handelt, erfolgt deren Berechnung gemäß $\$ 6,1 \mathrm{GOZ}$ analog. Der Zuschlag für Laseranwendung (GOZ-Nr. 0120) ist hier weder fachlich richtig noch gebührenordnungskonform anzusetzen. Die PT oder andere selbstständige Leistungen werden entweder einzeln durchgeführt oder können neben der Kürettage nach den BEMA-Nummern P200-P204 berechnet werden.

Wie auch in allen anderen Bereichen der Zahnheilkunde können Privatleistungen bei GKV-Versicherten nur dann berechnet werden, wenn die formalen Kriterien hinsichtlich der privat gewünschten Leistung eingehalten werden. Auch für die Parodontalbehandlung gilt die zwingend nötige Vereinbarung nach $\$ 4,5$ BMV-Z oder $₫ 7,7$ EKVZ, ohne die niemals eine Privatbehandlung beim GKV-Versicherten durchgeführt werden sollte.

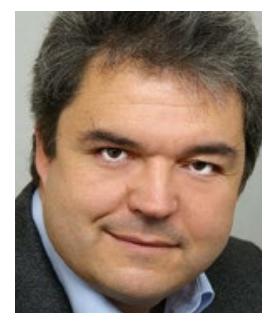

Dr. Dr. Alexander Raff

Mitglied im GOZ-Expertenrat des FVDZ 